\title{
XVII. Decas sexta novarum plantarum sueculentarum
}

\author{
Mr. A. H. Haworth
}

To cite this article: Mr. A. H. Haworth (1826) XVII. Decas sexta novarum

plantarum sueculentarum, Philosophical Magazine Series 1, 68:340, 125-132, DOI:

10.1080/14786442608674097

To link to this article: http://dx.doi.org/10.1080/14786442608674097

曲 Published online: 10 Aug 2009.

Submit your article to this journal $₫$

Џll Article views: 2

Q View related articles $₫$ 
purpose of showing the nature of the mutations which the theorem undergoes when we take the trapezium successively salient (fig. 8), re-entrant (fig. 9), and intersectant (fig. 10). The number of figures, however, which a complete exhibition of all the cases of the theorem would require, is not less than a hundred and twenty; perhaps even more, which have escaped my notice. These three figures are sufficient to show, also, that had we defined $\mathrm{AC}, \mathrm{FG}, \mathrm{DE}$, the three diagonals of the trapezium in fig. 8 , an elegant enunciation might be formed for the combination of them in pairs in reference to this theorem.

It is easy by a similar train of investigation to discover several analogous and equally beautiful properties of the trapezium: but as this paper has already exceeded the limits which I originally proposed to myself, I shall defer them to a future but not distant period. In conclusion, I shall just remark, that from the properties already given a series of interesting properties of the conic-sections are immediately deducible.

August 5, 1825.

XVII. Decas sexta novarum Plantarum Sueculentarim; Autore A. H. Haworth, Soe. Linn. Lond.-Soc. Horticult. Lond.-necnon Soc. Ces. Nat. Curios. Mosc. Socio, \&c. \&c.

To the Editor of the Philasophical Magazine and Journal. Sir,

HAVING just finished my sixth Decade of new Succulent 1 Plants, I lose no time in sending it for insertion in the next Number of your Magazine.

All the plants included in this decade, appear to me, after the most diligent research, to be entirely nondescript, and belong to the vast genus Mesembryanthemum, to which they form a conspicuous addition : for some of them are not only new as species, but constitute new and unrecorded sections. They are all flourishing and flowering in the royal and unrivalled gardens at Kew; and were sent thither by our most assiduous friend Bowie, whose adventurous and discriminating eye detected them growing spontaneously in the remote and arid regions of Southere Africa, whither this enterprising traveller is again about to repair. And he proposes to go not only with the intention of colleeting plants, seeds and roots, but also, as beretofore, in almast all the branches of animated nature.

And now, sir, most heartily wishing this extensive discoverer of succulent plants every possible success in his laborious 
undertakings, - a pleasant voyage to the Cape of Good Hope, a prosperous and excursive sojourn there, - a safe return to his native home, and a profitable termination to his interesting speculations; I remain, very respectfully, your old correspondent and friend,

Chelsea, Angust 1826.

A. H. Haworth.

Classis et Ordo. Icosandria Pentagrnia.

Genus, Mesendranthemum Auctorum.

Sectio (nova) Albinota, subacaulia : cæspitosa : radice perenni, foliis decussatis integris obliquè incurvis viridibus patulis crebrè albo tuberculatim magnipunctatis, infernè basive semiteretibus, supernè acinaciformiter triquetris vel ferè æquilateralibus, plùs minùs mucronulatis; floribus centralibus solitariis sessilibusluteis, staminibus erectopatentibus ut in sectione cui nomen Ringentia, anteque eam locanda.

albinotum. M. (keeled, white-dotted) foliis acinaciformiter sur-

1. sùm triquetris mucrone recurvulo punctisque subelevatis albicantibus sparsis.

Habitat C. B.S.

Florebat in regio horto Kewense, Sept. 1825. G. H. H... Anno priore ortum ex seminibus africanis a Domine Bowie collectis. Sequentia omnia quoque ejus assiduitati debemus.

Obs. Facies ferè M. aloöidis Nob. (absque ejus collectis staminibus).

Folia sub-biuncialia, latitudine 5-lineari, albicantibus punct is crebris quasi macularibus, et (certo situ) subindè sæpè squamulas pallidas simulantibus, rariùs viridilaus. Flos unus solùm vidi mediocris luteus ferè ut in M. felinum s. M. Lupinum Nob. at A. M. expansus, (nec vespere, basi bracteeis duabus magnis foliiformibus. Calyx 5-fidus, laciniis subæqualibus harum tribus obtusissimis aliquot brevioribus: duabus longè acutioribus parúmque longioribus. Corolla subsesquiuncialis, petalis latis apice crenulatis denticulatisve. Stamina brevissima lutea, antheris polliniferis saturatioribus cum luteo. Catera non examinavi.

albipunctum. M. (small-keeled white-dotted) foliis semitere-

2. tibus albo tuberculatim punctatis, supernè triquetris, mucronulo rufescente.

Habitut. C. B. S. 
Mr. Haworth's Sixth Decade of nero Succulent Plants. 127

Florebat in regio horto Kewense ad finem Sept. 1825. G. H. 4 .

Obs. Priori simile, at foliis duplò triplóve minoribus numerosioribus, a basi ad apicem sæpiùs attenuatis nec auctim carinatis seu subacinaciformibus ut in priore, punctis magis albis, sed flore (unico qui solùm vidi et non dissecavi) ut in illo.

\section{ß. majus, fere duplò.}

Sectio, Ringentia Nob. in Philos. Mag. Aug. 1824.

Ermininum. M. (The Ermine Chop) subacaule: glaucum: foliis

3. rugoso-magnipunctatis: marginibus supernè brevidentatis, carinâ integrâ.

Habitat C. B. S.

Florebat in regio horto Kewense, Maio 1825. G. H. 4 .

Descriptio. Herba densè cæspitosa. Folia subinde integra, at sæpiùs marginibus apicem versus 4-6-dentatis, dentibus validiusculis patentibus acutis, at sine setilâ finientibus. Flores solitarii terminales subsessiles absque bracteis. Calyx 5-fidus, infernè (cum germine) compresso-turbiniformis, superne foliolis oblongis æqualibus apice obtusis et obtusè subcarinatis, sed obsoletè et plùs minùs membranaceis, grossè tuberculato-punctatis foliorum more, at cum punctis oblongioribus. Corolla subuncialis ferè ut in $\boldsymbol{M}$. mustelino, vespere aperta, usque ad decimam horam (Augusto mense) petalis numerosis variè laxéque expansis capillaribus luteis calycinis foliolis 2 lineas superantibus. Filamenta longitudine valdè variantia, petalis semper duplò breviora lutea antheris longis cum polline subaurantiaco, infernè cum calyce altè coalita. Styli stigmatave 5 semilineam longi obtusi (lente intùs ramentacei) basi coaliti in unum stylum brevissimum subconicum pulposum. Germen incipiens depressum. Unum florem mense Septembre, vespere suaveolentem (at minùs gratum quàm $M$. mustelinum) solùm vidi.

ß. majus corollâ luteâ apice rubro.

Obs. Ante M. mirinum cui simile, certè locandum, et distinguitur foliorum crassiorum rugosiorum carinis integris, marginalibus dentibus brevioribus, absentiâque setulæ terminalis.

agnimum. M. (The Lamb's Chop) subacaule : canescens : punc-

4. tato-rugosulum: foliis semiteretibus puncto-serrulatis, subinde dentatis; basi intùs pustulatis. 
Habitat C. B. S.

Vigebat in regio horto Kewense, 1824, et florebat Maio 1826. G. H. 4.

Descriptio. Inter ejus affinia herba conspicua cæspitosa crassa et forsàn suæ sectionis. M. albidi habitus at 4-plò minùs, undique asperulum et ferè rugosulum punctịs elevatis numerosissimis. Folia sub-biuncialia, semunciam circiter lata decussata erecto-expansa, apice angustiora, at obtusa (junioribus obsoleto mucronulo) marginibus paulò post medium sub obsoletè obtuséque repando-dentatis, basi internè pustulâ magnâ albâ, et foliorum modo punctatâ. Florem unum sessilem, centralem, ebracteatum luteum perientem, ferè ut in $M$. mustelino, at longè majorem, (sed non magnum) solùm vidi. Inde locum speciei hujus apparet, et prope M. mustelinum locarem.

$\beta$. Paulò minus, foliorum dentibus obscurioribus. $\gamma$. foliis erectioribus integris.

Sectio, Anurca Nob. suffrutices vix semipedales, foliis subulatis teretiusculis sæpè incurvo-recurvulis, apicibus semper attenuatis aduncis ; floribus rubicundis antemeridianis, sæpiùs autumnalibus.

inconspicium. M. (small-flowered, rigid) prærigidum : foliis

5. trigono-semiteretibus parvis aduncis, floribus solitariis minutis terminalibus.

Habitat C. B. S.

Florebat in regio horto KewenseA.D.1825, autumno, rüsúmque Junio, \&c. 1826. G. H. $\psi$.

Descriptio. Suffrutex semipedalis dumosus, ramis patulis adscendentibus incurvisve filiformibus duris, junioribus lentis ope furvo-puberulis, apice ad solem coriuscantibus. Folia distincta semuncialia gracilia incurvata, et ad solem aëre aperto subpapuloso-micantia viridia, vix glaucescentia, apicibus attenuatis aduncis rufis, sive morientibus fulvis. Flores inconspicui subtrilineäres ebracteati, pedunculis brevissimis. Calyx 4-fidus ordinarius. Corolla antemeridiana saturatissimè rubicunda, petalis (ratione magnitudinis) latis obtusis, et forè semper intégris. Filamenta collecta, erecta, apice superneve saturatiora colore, antheris (ante anthesin) albis.

Florem unum solùm examindvi, sed alios varios vidi.

Sectio (nova) CrocèA. Suffruticès rärritilis secundis, foliis crassis glauco-cærulescentibus basi semi- 
Mr. Haworth's Sixth Decade of nero Succulent Plants. 129

teretibus, supernè obsoletè triquetris, floribus terminalibus solitariis minoribus vel mediocribus inodoris, primò sæpè luteis, denique sæpiùs croceis; pedunculis, calyceque inæquali, succulentis. Sectio, a sectione Sebacea separanda, quæ ultima gaudet ramulis decussatis, foliis teretiusculis crassioribus cærulescenti-glaucis, floribus umbellatis parvis suaveolentibus flavis mellinis.

Obs. Sectioni Crocea referenda sunt $M$. croceum Jacq. - M. purpuro-croceum Nob. et ejus var. flavocroceum (quæ var. nunc veram speciem propono) -atque etiam duæe sequentes, viz.

luteum. M. (upright yellow) foliis obtusis, floribus minoribus;

6. caule ramuloso erecto rigido, nodis tumentibus radiculigeris.

Habitat C. B. S.

Florebat in regio horto Kewense, Junio 1823, et per æstatem.

G. H. $々$.

Descriptio. M. flavo-croceo supradicto certè proximum, at elatius, ramosius, sed longè gracilius, ramis duris, foliis floribusque 3-plò minoribus.

In tertio anno erectum pedale ultráque est, ramorum nodis tumentibus terram versus (ope léntis) pullulantibus radiculis farctis. Folia erecta inter nodiis longiora lævia cærulescenti-glauca. Flores pauci solitarii, in summis ramorum altiorum terminales diurni lutei, pedunculis ebracteatis clavatis succulentis. Calyx 4phyllus, foliolis præinæqualibus ut in $M$. purpuro-croceo, duobus parvis membranatis: et 2, 4-plò majoribus emembranatis. Corolla lætè lutea, moriens saturatior. Filamenta erecta brevia antheraeque luteæ. Styli 8 ? minuti erecti, filamentis multoties breviores, pallidiores ovato-acuminati, per lentem ramentacei. Germen parvum pulposum 8-loculare.

luteolum. M. (acute-leaved, small yellow) foliis confertioribus 7. apice acuto recurvulo; ramis gracilibus densioribns, floribus parvis diurnis. M. lave Th. Prod.?Certissimè non Aitoni.

Habitat. C. B. S. . . . G. H. h.

Cum priore florebat in regio horto Kewense at quadrum plò humithas, ramis densioribus, longéque gracilioribus, nodis mimis tumentibus absque radiculis pullulantibus, et subindè flexuosè decumbentibus. . Folia paulò minora, confertiora, glauco-cærulea, at colore viridi palli- 
diorie. Flores numerosiores. In cretetis priore quadrat omninò.

$O b s$ s ab ultimo optimè distinguitar, foliorum apice acuto recurvulo, staturâque quadruplò minore.

Sectio Platyphylla, foliis ramulisve ferè semper plùs minùs ad solem papuloso-micantibus : radice subindè bienni annuâve.

clandestinum. M. (minute flowering Myrtle-leaved) humifu-

8. sum subpapulosum: foliis acutè ovatis petiolatis, petalis minutissimis.

Habitat C. B. S. . . . . G. H. 々.

Florcbat per æstatem in regio horto Kewense A.D. 1824.

Obs. Vix M. ovatum Thunb. Prod. p. 88, propter minutissimos fores, sed in cæteris fortè concordat. Non $M$. apetalum, ejusdem auctoris in loco, propter folia non lineari-lanceolata. Neque $M$. humifusum Aitoni, quod folia spatulata produxit.

Planta nostra est herba valdè foliosa parva sịve suffrutex, ramis numerosis herbaceis confertis procumbentibus 4-5 uncialibus teretibus, junioribus ad lncem papulosis, papulis (præcipuè foliorum) an lentem elevatis numerosissimis distinctis convexis atque ad solem sublimpidis. Internodii foliis breviores. Flores sapiùs terininales 1-3 nati pedunculo clavato tereti, folio breviore. Calyx 5-phyllus turbiniformis, foliolis subinæqualibus obtusis viridibus, apice (in aëre aperto) 1 ubentibus, duobus minoribus basi submembranaceis ut in plurimis. Corolla omnino clandestina petalis numerosis linearibus obtusis albis, calyce humilioribus, et ferè invisibilibus; sed lentis ope corolla ad solem antemeridianum incurvo-érectula et aliquantillúm apertula est, ubi inclusæe Antherce visæ sunt. Stamina brevissima alba, petalorum altitudine; antheris stramineis. Styli 5, ninutissimi acuminati. Capsula subturbinato-globosa píso minor supernè 5 -angularis, loculis totidem 5. Flores dúo solum examinavi.

Sectio Coraluina, Suffrutices læves erecti lignosi; ramis concinnè decussatis numeresis cortice phùs minùs cnstaneo; foliis semicylị̂draceis valdè glaucis: foribus terminalibus, magnis mediocribuske, sepissimè rubicundis.

lepidum. M. (pretty, white-flowered) foliis apice recurvulis 9. sub- 
submucronatis: foliolis calycum acuminatis patulis, post anthesin marcescentibus.

Habitat C. B. S. . . . . G, H. 々.

Florebat in regio horto Kewense, Aug. 1825.

Obs. Suffrutex erectus gracilis, secundo anno subpedalis, ramis ramulisve numerosis erectis. Folia ferè ut in $M$. producto. Nob. (cui simillimum ut ovum ovo) at fortè minùs incurvula. Flores majores antemeridiani candidi formosi, pedunculis longioribus. Styli 5. Capsula supernè latior quàm in M. producto, 5-locularis minúsque umbilicata, loculis totidem 5. Catera non examinavi.

$\mathrm{Ab}$ M. producto, cui proximum, calycum foliolis longè minoribus patentioribus perientibus, optimè distinguitur. Foliola calycina 2-3 in $M$. producto post florescentiam erecto-incurva carnosa auctaque persistunt.

Sectio Hispicaulia. Suffrutices dumosi subdodrantales caulibus pedunculisve plùs minùs hispidis : floribus antemeridianis rubicundis, rariúsve albis.

subcompressum. M. (slender upright twiggy) erecto-virga-

10. tum : foliis canescente-viridibus compresso-semiteretibus obliquè obtusissimis : ramis supremis parcè pilosulis.

Habitat C. B. S. . . . G. H. 々.

Vigebat in regio horto Kewense A.D. 1825, et Gorebat perparcè in Julio 1826.

Descriptio. Suffrutex subrigidus in secundo anno subpedalis gracilioribus ramis majisque virgatis quàm in affinibus, summis solùm pilis parvis respicientibus, cæeteris gradatim depilatis. Folia semper aliquantillùm compressa ad lucem cabescente-papuloso-micantia, ferè ut in $M$. candente Nob. à quo dignoscitur erectis ramis foliorumque formâ. Flores terminales solitarii violaceo-rubicundi vivacissimi (nec albi ut in M. candente) M. hispido duplò minores longéque pallidiores. Calyx 5-6-phyllus, foliolis duobus folifformibus majoribus obtusis, at nihilominùs valdè brevibus. Petala angusta. Filamenta antheraque albæ, petalis triplò breviores. Styli 6, arcuatim radianter recurvuli subulati lutei, filamentis breviores.-N. B. Florum pedunculi perbreves, et cum calyee plùs minùs gradatim crystallino-piłosuli, ut in affinibus, at per scalam omninò minorem. Flores duo solùm examinavi.

ß. minus. Varietatem ferè duplö minòrem et ferè de R 2 
pilatam ramis strictioribus vidi in ditissimo regio horto absque floribus. Fortè vera species. Simulat M. brevifolium parùm, at non effuse ramosum ut in illo, neque confertum.

XVIII. An Account of some Geological Specimens, collected by Captain P. P. KING, in his Survey of the Coasts of Australia, and by RoBert Brown, Esq., on the Shores of the Gulf of Carpentaria, during the Voyage of Captain Fuxnders. By William HenRy FitTon, M.D. F.R.S. V.P.G.S.

[Continued from p. 34.]

VI. A $S$ the superficial extent of Australia is more than A three-fourths of that of Europe, and the interior may be regarded as unknown*, any theoretic inferences, from the slight geological information hitherto obtained respecting this great island, are very likely to be deceitful; but among the few facts already ascertained respecting the northern portion of it, there are some which appear to afford a glimpse of general structure.

Captain Flinders, in describing the position of the chains of islands on the north-west coast of Carpentaria, Wessell's, the EnglishCompany's, and Bromby's Islands, remarks, that he had " frequently observed a great similarity both in the ground plans, and the elevations of hills, and of islands, in the vicinity of each other, but did not recollect another instance of such a likeness in the arrangement of clusters of islands.+ " The appearances which called for this observation, from a voyager of so much sagacity and experience in physical geography, must probably have been very remarkable; and, combined with information derivable from the charts, and from the specimens for which we are indebted to Captain King and Mr.

* The following are the proportions assigned by Captain de Freycinet to the principal divisions of the globe.-Vayage aux Terres Australes, p. 107.

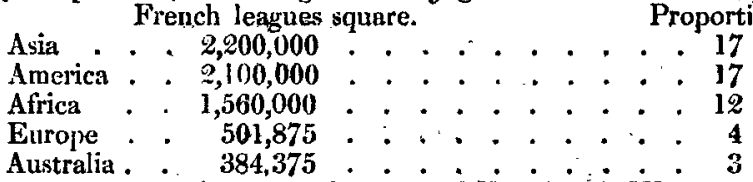

The most remote points from the coast of New South Wales, to which the late expeditions have penetrated, (and the interior hos never yet been examined in any other quarter, ) are not above 500 miles, in a direct line, from the sea; the average width of the island from east to west being more than 2000 miles, and from north to south more than 1000 miles.

+ Flinders, v. ii. p. 246 ; and Charts, Plates 14 and 15.-King's Charts, Plate 4. 